\title{
B2B predictive analytics: an untapped sector
}

\section{Opinion}

Much work in predictive analytics and data science has been primarily focused around the business to consumer sector (B2C). Certainly predictive analytics solutions have been applied to the B2B sector but it pales in comparison to what has been applied and learned in the $\mathrm{B} 2 \mathrm{C}$ sector. Yet, opportunities to gain more learning and knowledge exist where the same data science and data mining techniques can be applied to business to business (B2B). But there are some essential differences between the two sectors. In almost all cases, large businesses are typically excluded from any data mining/ data science exercise as the real data mining/data science opportunity is to improve the marketing effectiveness towards the small to medium size businesses. Another key difference is identifying the key contact or decision-maker within the organization. Since our marketing and data science efforts are directed at someone taking action, then the ability to take action on behalf of a given company can vary based on the contact person at that point in time. By exploring the key contact or decision-maker within the organization, we may want to better understand the specific characteristics and behaviors of the key contact along with the organization characteristics. But first, what kind of data is available for analysis? Can we capture job position and job role? In one exercise for a B2B software company, we analyzed job roles and job titles in terms of their response to a marketing offer. Differences were indeed identified by job title and job role and their impact on response. As well, we were also able to identify the prior engagement activity of contacts and how that may have impacted response. For example, we were able to determine the relationship between marketing response and the number of times a given contact is promoted prior to the marketing offer. Obviously, more information about how these contacts behave within the organization would have been useful in our analysis. But this is the challenge as B2B customers will only yield information to the software company that is relevant for billing purposes (i.e. job title and job role of contact) and not how contacts behave internally. Nevertheless, the overall modeling approach in developing predictive analytics solutions is identical between $\mathrm{B} 2 \mathrm{~B}$ and $\mathrm{B} 2 \mathrm{C}$.

Yet, even without this enriched contact information, B2B information has a significant advantage over B2C information in terms of available external information. In acquisition programs, external information is vital to the success of any analytics solutions. Within B2C exercises, most external information, particularly in the Canadian market is at an aggregate geographic level in terms of where people live. In other words, the demographic information appended to individuals essentially consists of averages, percentages, and median values at a geographic level. The notion here is that consumers in that specific geographic area comprise the same demographic profile. Meanwhile within the B2B sector, external data is at the individual company level which is a clear and distinct advantage in developing acquisition targeting tools for B2B marketers. Often referred to as firmographic information, variables pertaining to employee size, tenure of the company, sales, industry category, etc. are collected at the individual business level. With this powerful data, it is not unusual to see the targeting of B2B prospects yielding lift results of above 500 between the top decile and bottom decile which is clearly superior to what is traditionally seen in B2C acquisition programs.
Volume 6 Issue I - 2017

\author{
Richard Boire \\ Environics Analytics, Canada
}

Correspondence: Richard Boire, Environics Analytics, 33 Bloor Street East Suite 400, Toronto, ON M4W 3HI, Canada,

Email Richard.boire@environicsanalytics.ca

Received: January 05, 2017 | Published: September 06, 2017

Once a business becomes a customer, the existing data mining approach in dealing with existing customers is similar to what occurs for individual consumers. Type of customer, how long they have been a customer, where they are located, all represent demographics that are similar to what we observe with regular consumers. Meanwhile the spending behavior and campaign/contact behavior represent the rich robust behavior that will typically be used to predict the future behavior of that organization. Cross-sell, up sell, and retention models are all developed for existing business customers not unlike what is observed on customers who are individuals.

Yet as mentioned above, businesses do comprise 3 main categories based on size (large, medium and small) which marketers consider first before developing and marketing plan or strategy. Indeed it is the small business category where predictive analytics solutions offer the most significant benefits due to the huge volumes inherent in this sector. Yet, even within this sector, there is a sector of sole self-employed individuals who are quite unique from other small businesses. Although these individuals are mainly self-employed one person shops, one could argue that these businesses could be considered more like consumers. The same mechanics in understanding consumer individual behaviours and demographics is just as critical to understanding these self-employed entrepreneurs since more emphasis will be placed on their individual behaviours and information rather than the company as a whole in trying to predict future behaviour. Some practical examples from our experience are in the insurance and health sectors. In the insurance sector, tools can be developed to target brokers who are most likely to sell a given insurance company's products. However, the challenge in developing these tools is the source data that is available in creating the analytical file. The information collected on brokers by many insurance companies tends to be sparse and inconsistent as broker data is collected from multiple sources. Another similar example of this type of challenge are sales persons/agents where once again sales agents can work for multiple organizations and data is again being collected in multiple places. The data challenge is trying to pool broker or sales data across multiple databases in order to create that one unified view of the broker or salesperson. It is this unified view of the sales person or broker that allows us to develop strategies for both high performers and high potential performers.

Within the health sector, rich individual level data for doctors exists through organizations such as IMS. Examples of this doctorlevel data include type of specialization, university attended, tenure as a doctor, script behavior, etc. This can be extremely valuable to pharmaceutical companies as they can now develop predictive models 
which identify doctors who are most likely to prescribe a certain drug. Based on the type of solution, resources can then be better allocated to deliver higher ROI on a particular marketing/sales initiative.

Another challenge in dealing with businesses is the treatment of head office vs. branch offices. For instance, should branch offices be treated as separate marketing entities as opposed to head offices only. This may not be totally relevant for small businesses as most of them would only have one office. But nevertheless, there will be some small businesses that have multiple offices where again true understanding of the specific marketing objectives will dictate the appropriate level of treatment.

The process of developing B2B predictive models is no different than $\mathrm{B} 2 \mathrm{C}$ models. The significant difference is the domain knowledge in dealing with data that is unique to businesses such as firmographic information and self-employed entrepreneurs such as doctors, insurance brokers, and sales agents. The literature in predictive analytics often deals with consumers with the development of models in the B2B environment not garnering much attention. This scenario limits our ability to gain knowledge as practitioners of data science/ data mining since there is a whole body of work and practices that have occurred within this sector. As practitioners, we should encourage the sharing of this work and learning. Practical experience in the B2B sector does provide unique levels of experience and ultimately learning beyond the $\mathrm{B} 2 \mathrm{C}$ sector but which would be invaluable for those practitioners who are making the leap from the $\mathrm{B} 2 \mathrm{C}$ world to the B2B world.

\section{Acknowledgements}

None.

\section{Conflict of interest}

The author declares no conflict of interest. 